\title{
Correction to: Fundamentals of Brooks-Iyengar Distributed Sensing Algorithm
}

\section{Correction to:}

P. Sniatala et al., Fundamentals of Brooks-Iyengar Distributed Sensing Algorithm, https://doi.org/10.1007/978-3-030-33132-0

This book was inadvertently published with an incorrect affiliation of the author

"M. Hadi Amini". The affiliation has been corrected now and it reads as follows:

M. Hadi Amini

School of Computing and Information Sciences, Florida International University, Miami, USA. 